\title{
A review of Rheocricotopus (Psilocricotopus) chalybeatus species group from China, with the description of three new species (Diptera, Chironomidae)
}

\author{
Wenbin Liu ${ }^{1, \dagger}$, Xiaolong Lin ${ }^{1, \ddagger}$, Xinhua Wang ${ }^{1, \S}$ \\ I College of Life Science, Nankai University, Tianjin 300071, China \\ † http://zoobank.org/BA99D0CE-0E18-46EF-BB3B-061CDE4A25B9 \\ ¥ http://zoobank.org/AC3964DC-0709-4DCB-96AA-6EBEDFA04C07 \\ § http://zoobank.org/ACC3483B-5DD1-4F2D-88F7-1CE4C6458B68 \\ Corresponding author: Author (xhwang@nankai.edu.cn)
}

Academic editor: V. Blagoderov | Received 26 September 2013 | Accepted 21 February 2014 | Published 12 March 2014

http://zoobank.org/DC6CCFBE-6D47-4753-B95F-D44E0B80B511

Citation: Liu W, Lin X, Wang X (2014) A review of Rheocricotopus (Psilocricotopus) chalybeatus species group from China, with the description of three new species (Diptera, Chironomidae). ZooKeys 388: 17-34. doi: 10.3897/zookeys.388.6316

\begin{abstract}
The Rheocricotopus (Psilocricotopus) chalybeatus species group from China is reviewed. Three new species, $R$. (P.) brochus sp. n., $R$. (P.) rotundus sp. n. and $R$. (P.) serratus sp. n. are described as adult males. $R$. $(P$.) imperfectus Makarchenko \& Makarchenko, 2005, $R$. (P.) robacki (Beck \& Beck, 1964) and $R$. (P.) valgus Chaudhuri \& Sinharay, 1983 are recorded from China for the first time and annotated. The diagnosis for the species group is emended and a key to adult males of the species group in China is presented.
\end{abstract}

\section{Keywords}

Chironomidae, Rheocricotopus, chalybeatus species group, new species, key, China

\section{Introduction}

The genus Rheocricotopus was erected by Thienemann and Harnisch (1932). Originally, Edwards (1929) treated it as a group of the genus Spaniotoma Philippi, 1865. Brundin (1956) reaffirmed the valid generic status of Rheocricotopus to be followed by Lehmann (1969) and other workers. It can be separated from other orthoclad genera by the following combination of characters: hairy eyes; without dorsomedial extension;

Copyright Wenbin Liu et al. This is an open access article distributed under the terms of the Creative Commons Attribution International License (CC BY 4.0), which permits unrestricted use, distribution, and reproduction in any medium, provided the original author and source are credited. 
developed pulvilli; acrostichals beginning near antepronotum; plate-like superior volsella and pointed anal point with posterolaterally directed setae. The immature stages of Rheocricotopus can be collected in streams and rivers, rarely in the littoral zone of lakes (Cranston et al. 1989). So far, 70 species (Ashe and O'Connor 2012) were recorded in all zoogeographic regions in the world.

Sæther (1985) reviewed the genus Rheocricotopus Thienemann \& Harnisch, 1932 in the world and divided the genus into two subgenera (Rheocricotopus sensu stricto and Psilocricotopus Sæther) including six species groups (atripes species group, chalybeatus species group, godavarius species group, tuberculatus species group, fuscipes species group and effusus species group). Wang and Sæther (2001) erected orientalis, a new species group. The Rheocricotopus chalybeatus species group can be distinguished from other species groups by the following combination of characters: gonostylus either with pronounced, preapical, triangular crista dorsalis or with apically sharp upward bend fused with apparent crista dorsalis; humeral pit moderately large, ovoid or circular, if large and somewhat rectangular gonostylus bent sharply upwards distally; superior volsella rounded, relatively small, never with projection. To date, 22 species were recorded in the chalybeatus species group (Sxther 1985; Caspers 1987; Chaudhuri and Sinharay 1983; Hazra and Chaudhuri 2004; Johannsen 1932; Makarchenko and Makarchenko 2005; Sasa 1990, 1991; Sasa and Suzuki 2000; Wang and Zheng 1989, 1991; Wang et al. 2004).

In China, 6 species of chalybeatus species group $[R$. (P.) emeiensis Wang \& Zheng, 1989, R. (P.) nigrus Wang \& Zheng, 1989, R. (P.) bifasciatus Wang \& Zheng, 1991, $R$. (P.) brachypus Wang \& Zheng, 1991, $R$. (P.) chalybeatus (Edwards, 1929) and $R$. (P.) taiwanensis Wang et al., 2004] had been recorded (Wang 2000, Wang et al. 2004).

Based on specimens from China, in this paper, three new species are described, and a key to the Chinese species of chalybeatus group is presented.

\section{Materials and methods}

The morphological nomenclature follows Sæther (1980). The material examined was mounted on slides following the procedures outlined by Sæther (1969). The specimens examined in this study are deposited in the College of Life Sciences, Nankai University, China.

\section{Taxonomy}

Rheocricotopus (Psilocricotopus) bifasciatus Wang \& Zheng, 1991 http://species-id.net/wiki/Rheocricotopus_bifasciatus

Rheocricotopus bifasciatus Wang \& Zheng, 1991: 100.

Rheocricotopus (Psilocricotopus) bifasciatus Wang, 2000: 639, Ashe and O'Connor 2012: 560. 
Specimens examined. Type material: Holotype, $\hat{\jmath}$, Chongqing City, Jinfo Mountain, 2901'90"N, 107'16'20"E, 9.v.1986, sweeping, Wang XH. Paratype (1): 10 , as holotype.

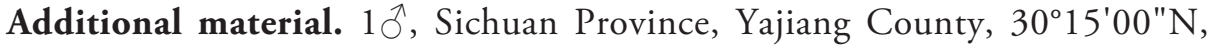
$101^{\circ} 02^{\prime} 00^{\prime \prime E}, 14 . v i i .1997$, sweeping, Wang XH; $10^{\lambda}$, Hunan Province, Taoyu-

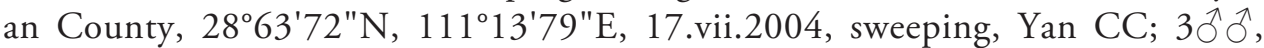
Ningxia Hui Autonomous Region, Jingyuan County, 35⒍'33"N, 106 29'08"E, 7.viii.1987, sweeping, Wang XH; 10 , Ningxia Hui Autonomous Region, Jin-

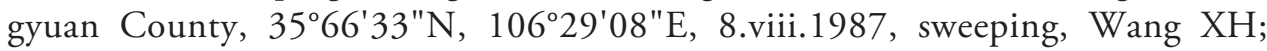

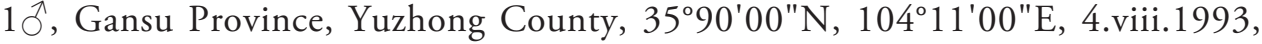
sweeping, Bu WJ.

Diagnosis. This species can be separated from other members of the group by the following combination of characters: tergites I, II and IV pale brown, others dark brown; AR 0.90; wing anal lobe reduced; humeral pit large, ovoid; Costal extension $83 \mu \mathrm{m}$ long.

Remarks. The additional specimens are similar to the description of Wang and Zheng (1991). The species is recorded from Palearctic Region for the first time.

Distribution. China (Chongqing Municipality, Sichuan, Hunan and Gansu Provinces, Ningxia Hui Autonomous Region).

\section{Rheocricotopus (Psilocricotopus) brachypus Wang \& Zheng, 1991}

http://species-id.net/wiki/Rheocricotopus_brachypus

Rheocricotopus brachypus Wang \& Zheng, 1991: 101.

Rheocricotopus (Psilocricotopus) brachypus Wang, 2000: 639, Ashe and O'Connor 2012: 560.

Specimens examined. Type material: Holotype, $\widehat{\partial}$, Guangdong Province, Fengkai County, Heishiding National Nature Reserve, 2330'02"N, $111^{\circ} 55^{\prime} 01^{\prime \prime E}, 12$. iv. 1985 , sweeping, Wang XH.

Additional material. $10^{\lambda}$, Hubei Province, Hefeng County, $29^{\circ} 91^{\prime} 00^{\prime \prime} \mathrm{N}$, $110^{\circ} 03^{\prime} 00^{\prime E}$, 16.vii.1999, light trap, Ji BC; $10^{\top}$, Sichuan Province, Yajiang County, $30^{\circ} 15^{\prime} 00^{\prime \prime} \mathrm{N}, 101^{\circ} 02^{\prime} 00^{\prime} \mathrm{E}, 14 . v i i .1997$, sweeping, Wang XH; $1 \mathrm{O}^{\top}$, Xizang Autonomous Region, Bayi County, Shergmla Mountain, 2964'07"N, 94³6'01"E, 28-30. ix.1997, yellow trap, Solhøy T \& Skartveit J.

Diagnosis. This species can be separated from other members of the group by the following combination of characters: AR 0.43; anal lobe developed; anal point robust.

Remarks. Wang and Zheng (1991) described this species without humeral pit which as diagnostic characteristic. However, after examining the holotype, we find a medium, relatively shallow, ovoid humeral pit existing.

Distribution. Oriental China (Guangdong, Hubei and Sichuan Provinces, Xizang Autonomous Region). 


\section{Rheocricotopus (Psilocricotopus) brochus sp. $\mathrm{n}$.}

http://zoobank.org/74D7F9E8-E9E8-4BE0-A21B-DDEB23C88D8B

http://species-id.net/wiki/Rheocricotopus_brochus

Figs 1-6

Diagnosis. The adult male can be distinguished from known species of the species group and the genus by the following combination of characters: crista dorsalis toothliked; tergites I, II and IV yellow, tergite III mainly yellow with a brown circular area, other tergites brown.

Description. Male imago $(\mathrm{n}=12)$

Total length $1.75-2.70,2.18 \mathrm{~mm}$. Wing length $1.25-1.60,1.33 \mathrm{~mm}$. Total length/ wing length $1.35-1.78,1.68$. Wing length/length of profemur 1.76-2.31, 2.04 .

Coloration. Head and thorax brown. Tergites (Fig. 1) I, II and IV yellow, tergite III mainly yellow but having a brown circular area, other tergites brown.

Head. AR 0.63-0.89, 0.72. Ultimate flagellomere 245-360, $284 \mu \mathrm{m}$ long. Temporal setae 4-7, 5, including 1-3, 2 inner verticals; $1-3,2$ outer verticals and $0-2,1$ postorbital. Clypeus with 7-10, 8 setae. Cibarial pump, tentorium and stipes as in Fig. 2. Tentorium 75-140, $123 \mu \mathrm{m}$ long, 13-25, $19 \mu \mathrm{m}$ wide. Stipes 100-125, $118 \mu \mathrm{m}$ long, 8-10, $8 \mu \mathrm{m}$ wide. Palpomere lengths (in $\mu \mathrm{m}$ ): 20-25, 23; 38-55, 47; 90-130, 105; 110-138, 120; 205-250, 232. L: $5^{\text {th }} / 3^{\text {rd }} 1.92-2.67,2.23$. Third palpal segment with 2 sensilla clavata.

Wing (Fig. 3). Anal lobe reduced. VR 1.06-1.13, 1.07. Costal extension 45-65, $51 \mu \mathrm{m}$ long. Brachiolum with 1 seta. $\mathrm{R}$ with $3-6,5$ setae. Remaining veins bare. Squama with 1-2, 2 setae.

Thorax (Fig. 4). Antepronotum with 2-4, 4 lateral setae. Dorsocentrals 5-7, 6; acrostichals 9-13, 11; prealars 2-3, 3. Scutellum with 2-4, 4 setae. Humeral pit moderately large, ovoid.

Legs. Spur of fore tibia 23-40, $35 \mu \mathrm{m}$ long; spurs of mid tibia 13-25, $15 \mu \mathrm{m}$ and $10-18,12 \mu \mathrm{m}$ long; spurs of hind tibia $38-50,41 \mu \mathrm{m}$ and $10-20,15 \mu \mathrm{m}$ long. Hind tibial comb with 9-12, 10 spines, $20-48,31 \mu \mathrm{m}$ long. Width at apex of fore tibia

Table I. Lengths (in $\mu \mathrm{m}$ ) and proportions of legs of $R$. (P.) brochus sp. n.

\begin{tabular}{c|c|c|c}
\hline & $\mathrm{P}_{1}$ & $\mathrm{P}_{2}$ & $\mathrm{P}_{3}$ \\
\hline $\mathrm{fe}$ & $530-924,662$ & $530-800,713$ & $570-820,629$ \\
\hline $\mathrm{ti}$ & $640-860,713$ & $530-690,670$ & $660-870,724$ \\
\hline $\mathrm{ta}_{1}$ & $570-770,618$ & $330-450,362$ & $410-550,446$ \\
\hline $\mathrm{ta}_{2}$ & $310-420,343$ & $150-200,161$ & $180-260,205$ \\
\hline $\mathrm{ta}_{3}$ & $210-300,240$ & $100-130,110$ & $150-210,169$ \\
\hline $\mathrm{ta}_{4}$ & $150-200,163$ & $50-120,63$ & $70-100,83$ \\
\hline $\mathrm{ta}_{5}$ & $70-95,81$ & $55-70,57$ & $60-80,69$ \\
\hline $\mathrm{LR}$ & $0.83-0.90,0.88$ & $0.61-0.65,0.63$ & $0.60-0.63,0.61$ \\
\hline $\mathrm{BV}$ & $2.32-2.52,2.41$ & $3.94-4.62,4.10$ & $3.30-3.49,3.42$ \\
\hline $\mathrm{SV}$ & $2.07-2.32,2.20$ & $3.11-3.48,3.26$ & $2.95-3.07,3.02$ \\
\hline $\mathrm{BR}$ & $2.00-4.00,2.98$ & $1.57-3.00,2.51$ & $2.25-3.25,2.67$ \\
\hline
\end{tabular}



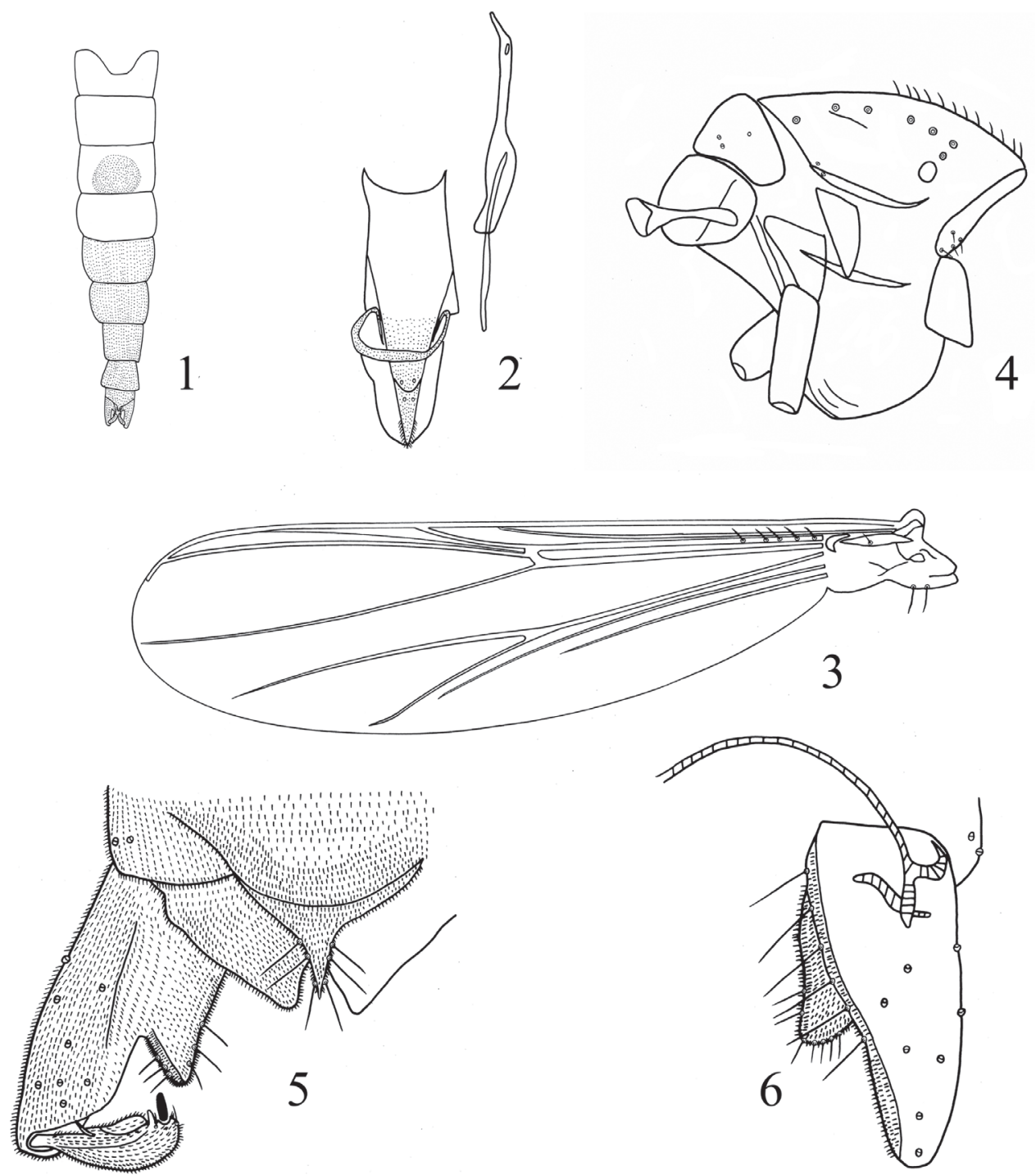

Figures I-6. Rheocricotopus (Psilocricotopus) brochus sp. n., male. I abdomen tergites coloration $\mathbf{2}$ cibarial pump, tentorium and stipes $\mathbf{3}$ wing $\mathbf{4}$ thorax $\mathbf{5}$ hypopygium (dorsal view) $\mathbf{6}$ hypopygium (ventral view).

35-45, $41 \mathrm{~mm}$, of mid tibia 33-45, $41 \mathrm{~mm}$, of hind tibia 30-45, $38 \mathrm{~mm}$. Lengths (in $\mu \mathrm{m})$ and proportions of legs as in Table 1.

Hypopygium (Figs 5-6). Anal point tapering to apex, 33-41, $37 \mu \mathrm{m}$ long, 25-50, $37 \mu \mathrm{m}$ wide in base, with 3-4, 4 lateral setae in each side. Laterosernite IX with 1-3, 2 setae. Phallapodeme 35-68, $55 \mu \mathrm{m}$ long. Transverse sternapodeme 35-88, $59 \mu \mathrm{m}$ long. Gonocoxite 138-191, $157 \mu \mathrm{m}$ long. Superior volsella triangular, 20-40, $31 \mu \mathrm{m}$ long, with 5-8, 7 setae. Gonostylus 60-75, $66 \mu \mathrm{m}$ long. Megaseta 9-13, $11 \mu \mathrm{m}$ long. Crista dorsalis tooth-shaped. HR 2.11-2.60, 2.38. HV 2.59-3.60, 3.31. 
Table 2. Main differences between $R$. (P.) brochus sp. n., $R$. (P.) bifasciatus and $R$. (P.) insularis.

\begin{tabular}{c|c|c|c}
\hline & $\boldsymbol{R} .(\boldsymbol{P}$.$) brochus sp. \mathbf{n}$. & $\boldsymbol{R} .(\boldsymbol{P}$.$) bifasciatus$ & $\boldsymbol{R}$. (P.) insularis \\
\hline AR & $0.63-0.89,0.72$ & 0.90 & $0.71-0.74$ \\
\hline Length of costal extension & $45-65,51 \mu \mathrm{m}$ & $83 \mu \mathrm{m}$ & $96 \mu \mathrm{m}$ \\
\hline Squama & $1-2,2$ setae & 2 setae & 8 setae \\
\hline Shape of humeral pit & medium, oviod & large, rounded & oviod \\
\hline Tergite coloration & $\begin{array}{c}\text { TI, II, IV yellow, TIII with a } \\
\text { brown circular area, others brown }\end{array}$ & $\begin{array}{c}\text { TI, II, IV yellow, } \\
\text { others brown }\end{array}$ & all tergites brown \\
\hline \multicolumn{4}{|r}{}
\end{tabular}

Type material. Holotype: $\widehat{\partial}$ (BDN. C11A32), China, Zhejiang Province, Yueqing City, Lingdi County, Jiulong Village, 28 31'00"N, 120 $96^{\circ} 00^{\prime \prime E}$, 18.iv.2011,

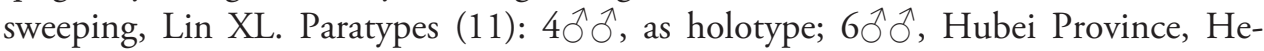
feng County, $29^{\circ} 91^{\prime} 00^{\prime \prime N}, 110^{\circ} 03^{\prime} 00^{\prime \prime E}, 16 . v i i .1999$, light trap, Ji BC; $10^{\top}$, Jiangxi

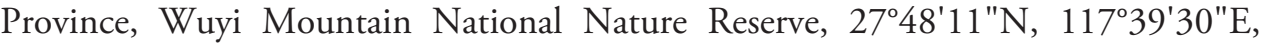
13.vi.2004, light trap, Yan CC.

Etymology. The specific name is an adjective, from Latin brochus, meaning tooth, referring to tooth-shaped crista dorsalis.

Remarks. The new species resembles $R$. (P.) bifasciatus Wang \& Zheng, 1991 and $R$. (P.) insularis Makarchenko \& Makarchenko, 2005 in the following combination of characters: anal point long, pointed distally; wing anal lobe reduced; crista dorsalis tooth-shaped. But the new species can be separated from the latter species in the basis following combination of characters in Table 2.

Female and immature stages unknown.

\section{Rheocricotopus (Psilocricotopus) chalybeatus (Edwards, 1929)}

http://species-id.net/wiki/Rheocricotopus_chalybeatus

Spaniotoma chalybeatus Edwards, 1929: 331.

Eukiefferiella urbanus Goetghebuer, 1932: 101.

Trichocladius lerutbi Goetghebuer, 1939: 2.

Rheocricotopus chalybeatus Lehmann, 1969: 354; Hirvenoja 1973: 340; Langton 1984: 98.

Rheocricotopus (Psilocricotopus) chalybeatus Sæther, 1985: 82; Wang 2000: 639, Ashe and O'Connor 2012: 561.

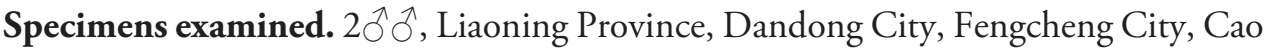

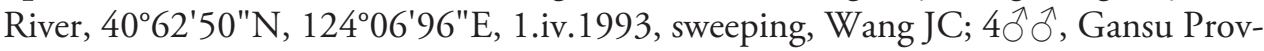

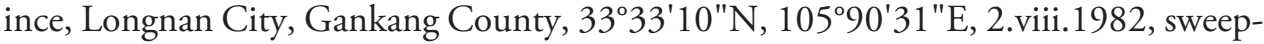

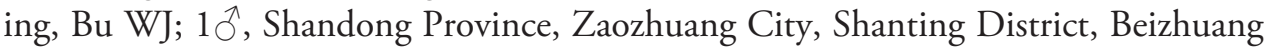

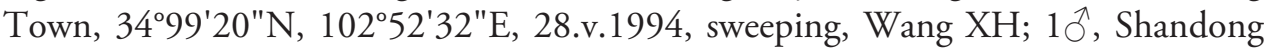
Province, Yantai City, Muping District, 37³8'62"N, 121 ${ }^{\circ} 59^{\prime} 57^{\prime \prime E}$ 28.viii.1988, sweeping, Li HY; $1{ }^{\lambda}$, Shaanxi Province, Baoji City, Feng County, Tsinling Moun- 


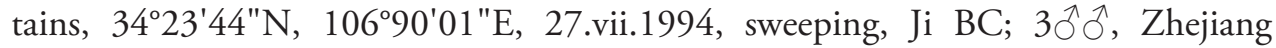
Province, Wenzhou City, Yueqing City, Furong Town, 33⒍'03"N, $121^{\circ} 02^{\prime} 73^{\prime \prime E}$, 2.viii.2010, light trap, Lin XL.

Diagnosis. This species can be separated from its congeners by the following combination of characters: AR 0.89-1.15; $\mathrm{R}$ with 2-4 setae; squama with 8-14 setae; Costa not produced or scarcely produced.

Remarks. The additional specimens mainly agree with the description of Lehmann and other workers. But costal extension of specimens from Oriental Region (35-40 $\mu \mathrm{m}$ long) longer than from Palearctic Region (0-15 $\mu \mathrm{m}$ long).

Distribution. China (Liaoning, Gansu, Shandong, Shaanxi and Zhejiang Provinces), Algeria, Balearic Islands, Belarus, Belgium, Corsica, Czech Republic, Denmark, Finland, France, Germany, Great Britain, Hungary, Ireland, Italy, Japan, Lebanon, Luxembourg, Mongolia, Morocco, Netherlands, Poland, Portugal, Romania, Russia, Slovakia, Spain, Switzerland, Syria, Tunisia, Turkey, Ukraine.

\section{Rheocricotopus (Psilocricotopus) emeiensis Wang \& Zheng, 1989}

http://species-id.net/wiki/Rheocricotopus_emeiensis

Rheocricotopus emeiensis Wang \& Zheng, 1989: 311.

Rheocricotopus (Psilocricotopus) emeiensis Wang, 2000: 639, Ashe and O'Connor 2012: 563.

Specimens examined. Type material: Holotype, $\widehat{\partial}$, Sichuan Province, Leshan City, Emei Mountain, 2958'18"N, 103²9'15"E, 17.v.1986, sweeping, Wang XH.

Additional material. $1 \hat{\jmath}$, Xinjiang Uygur Autonomous Region, Haba County,

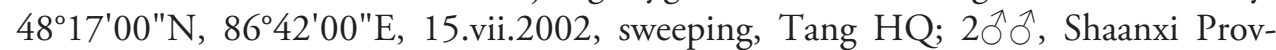
ince, Hanzhong City, Liuba County, Xiaoliuba Village, 33⒍'03"N, 106 90'31"E,

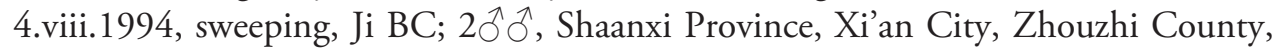

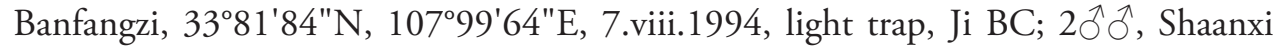
Province, Xi'an City, Zhouzhi County, Banfangzi, 3381'84"N, 10799'64"E, 10.viii.1994, light trap, Ji BC; $1 \widehat{\text { }}$, Yunnan Province, Kunming City, Fumin County, $25^{\circ} 22^{\prime} 61^{\prime \prime} \mathrm{N}, 102^{\circ} 52^{\prime} 32^{\prime \prime E}$, 1.vi.1996, sweeping, Wang XH; $10^{\wedge}$, Yunnan Province,

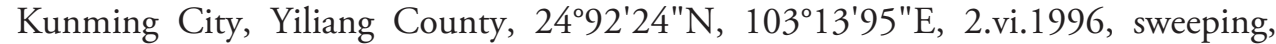
Wang XH; $10^{\lambda}$, Fujian Province, Xiamen City, 2448'24"N, 11808'44"E, 15.v.1993, sweeping, Bu WJ; 10, Guizhou Province, Guiyang City, 2660'17"N, 106 $70^{\prime} 36^{\prime \prime} \mathrm{E}$,

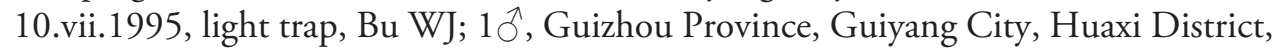
264'34"N, 10666'66"E, 23.vii.1995, sweeping, Bu WJ.

Diagnosis. This species can be separated from other members of the group by the following combination of characters: $\mathrm{R}$ bare; anal point short, pointed distally.

Remarks. The additional specimens are similar to the description of Wang and Zheng (1989). The species is recorded from Palearctic Region for the first time.

Distribution. China (Fujian, Guizhou, Sichuan, Shaanxi and Yunnan Provinces, Xinjiang Uygur Autonomous Region). 
Rheocricotopus (Psilocricotopus) imperfectus Makarchenko \& Makarchenko, 2005 http://species-id.net/wiki/Rheocricotopus_imperfectus

Rheocricotopus (Psilocricotopus) imperfectus Makarchenko \& Makarchenko, 2005: 126;

Makarchenko and Makarchenko 2011: 120, Ashe and O'Connor 2012: 564.

Specimens examined. $10^{\lambda}$, Hubei Province, Shennongjia Forest Region, 31 $1^{\circ} 74^{\prime} 56^{\prime \prime N}$, 11067'53"E, 19.vii.1997, sweeping, Du YZ; 10̄, Hubei Province, Lichuan City,

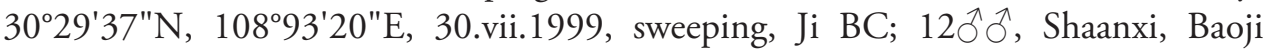
City, Feng County, Tsinling Mountains, 34²3'44"N, 106 90'01"E, 28-30.vii.1994, sweeping, Bu WJ; 1 $\widehat{\jmath}$, Shaanxi, Ankang City, Ningshan County, Huoditang Town,

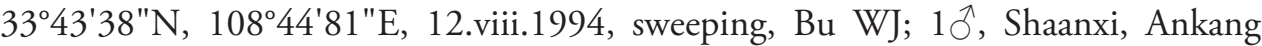
City, Ningshan County, Xunyangba Town, 3354'82"N, 10854'77"E, 17.viii.1994,

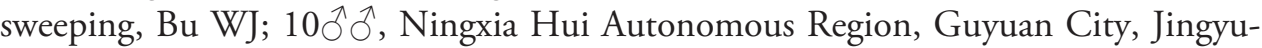
an County, Liupan Mountain, 35 $78^{\prime} 97^{\prime \prime N}, 106^{\circ} 28^{\prime} 93 " \mathrm{E}, 6-7 . v i i i .1987$, sweeping, Wang XH.

Diagnosis. This species can be separated from other members of the group by the following combination of characters: AR 0.47; humeral pit large and rounded; acrostichal absent; anal point of hypopygium sharply triangular, with 10 setae along the edges; gonostylus slightly curved, with roundish triangular crista dorsalis.

Remarks. Chinese specimens mainly agree with the description of Makarchenko and Makarchenko (2005), but Chinese specimens with more setae in R (4-8) than the specimens in Russia ( $\mathrm{R}$ with 3 setae).

Distribution. China (Hubei and Shaanxi Provinces, Ningxia Hui Autonomous Region), Russia (Far East).

\section{Rheocricotopus (Psilocricotopus) nigrus Wang \& Zheng, 1989}

http://species-id.net/wiki/Rheocricotopus_nigrus

Rheocricotopus nigrus Wang \& Zheng, 1989: 311.

Rheocricotopus (Psilocricotopus) nigrus Wang, 2000: 639; Makarchenko and Makarchenko 2011: 120, Ashe and O'Connor 2012: 566.

Specimens examined. Type material: Holotype, $\widehat{\partial}$, Hubei Province, Xiangyang

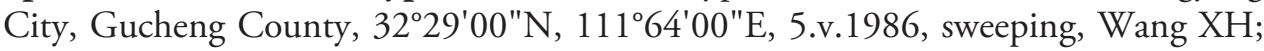
Paratype (1): $10^{\lambda}$, as holotype.

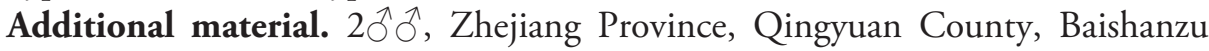
National Nature Reserve, 27ำ'23"N, $119^{\circ} 19^{\prime} 06^{\prime \prime E}$, 18.iv.1995, sweeping, Ji BC;

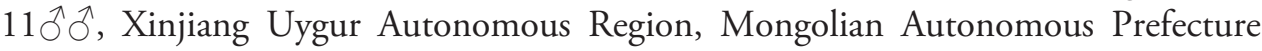

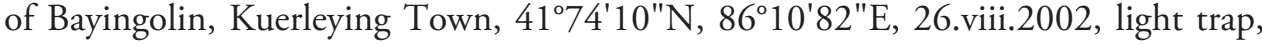
Tang HQ; $10^{\lambda}$, Xinjiang Uygur Autonomous Region, Mongolian Autonomous Pre-

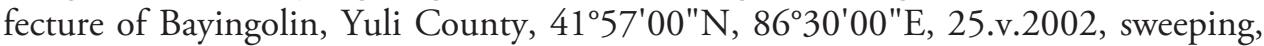
Tang HQ; $10^{\lambda}$, Xinjiang Uygur Autonomous Region, Haba County, $48^{\circ} 17^{\prime} 00^{\prime \prime N}$, 
86²'00"E, 15.vii.2002, sweeping, Tang HQ; 1ふ̄, Fujian Province, Nanping City,

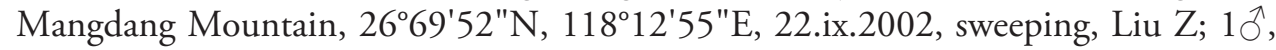
Fujian Province, Nanping City, Mangdang Mountain, 26⒍'52"N, 118¹2'55"E, 23.ix.2002, sweeping, Liu Z.

Diagnosis. The adult male can be separated from other members of the group by the following combination of characters: body totally dark brown; AR 1.30; dorsocentrals 20; humeral pit large, similar to the square.

Remarks. The species distributed in both Oriental and Palearctic Region. The specimens from Palearctic Region have fewer dorsocentrals (13-17) than those from Oriental Region (20).

Distribution. China (Hubei, Zhejiang and Fujian Provinces, Xinjiang Uygur Autonomous Region), Russia (Far East).

\section{Rheocricotopus (Psilocricotopus) robacki (Beck \& Beck, 1964)}

http://species-id.net/wiki/Rheocricotopus_robacki

Tricocladius robacki Beck \& Beck, 1964: 204.

Rheocricotopus kenorensis Sæther, 1969: 88.

Rheocricotopus (Psilocricotopus) robacki Sæther, 1985: 79, Ashe and O'Connor 2012: 567.

Specimens examined. $1 \hat{\jmath}$, Shaanxi Province, Xi'an City, Zhouzhi County, Banfangzi,

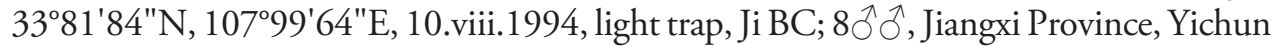

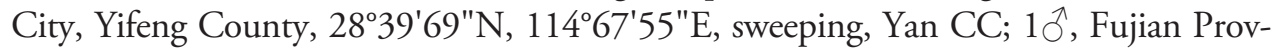
ince, Longyan City, Shanghang County, 2505'21"N, 11641'52"E, 6.v.1993, sweeping, Wang XH; $2 \hat{\jmath} \widehat{\jmath}$, Xinjiang Uygur Autonomous Region, Boertala Mongol Autonomous

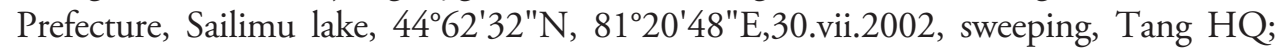

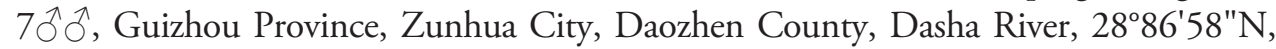

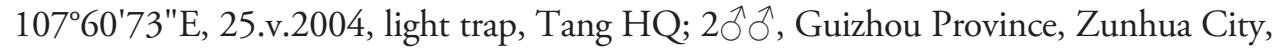
Daozhen County, Dasha River, 2886'58"N, 10760'73"E, 24.viii.2004, sweeping, Yu X; $3 \widehat{\jmath}$, Yunnan Province, Dali Bai Autonomous Prefecture, Eryuan County, Niujie Town, 262 $25^{\prime} 55^{\prime \prime} \mathrm{N}, 99^{\circ} 98^{\prime} 90^{\prime \prime E}$, light trap, Wang BX; $1 \delta^{\lambda}$, Tibet, Xigaze, Nielamu

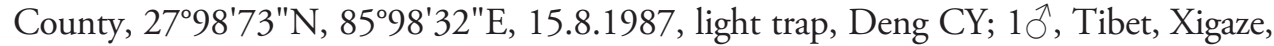
Nielamu County, $27^{\circ} 98^{\prime} 73^{\prime \prime} \mathrm{N}, 85^{\circ} 98^{\prime} 32^{\prime \prime E}, 21.9 .1987$, light trap, Deng CY.

Diagnosis. The species is characterized by having a relatively high AR (1.14-1.24), very weak and short acrostichals, 8-14 dorsocentrals, reduced number of bristles on squama, anal tergite extending beyond tip of anal point, superior volsella triangular, crista dorsalis triangular, apex pointed.

Remarks. Chinese specimens mainly agree with the description of Sæther (1969, 1985). It is recorded in Palearctic Region for the first time. Chinese specimens have lower body length $(2.55-3.13 \mathrm{~mm})$ and lower AR 1.07 than species from Nearctic Region (total length 3.10-3.30 mm, AR 1.18).

Distribution. China (Fujian, Guizhou, Jiangxi, Shaanxi and Yunnan Provinces, Xinjiang Uygur Autonomous Region, Tibet), Canada, U.S.A. 


\section{Rheocricotopus (Psilocricotopus) rotundus sp. $\mathbf{n}$.}

http://zoobank.org/5796E5A3-6914-462C-97E2-9CE1709E522E

http://species-id.net/wiki/Rheocricotopus_rotundus

Figs $7-11$

Diagnosis. The adult male of the new species can be distinguished from known species of the genus by the following combination of characters: low AR 0.25-0.29; superior volsella rounded.

Description. Male $(\mathrm{n}=2)$.

Total length $1.58-1.98 \mathrm{~mm}$. Wing length $0.86-1.20 \mathrm{~mm}$. Total length/wing length 1.66-1.84. Wing length/length of profemur 1.74-2.61.

Coloration. Head and abdomen yellow brown, thorax without distinct pattern.

Head. Antenna as in Fig. 7. AR 0.25-0.29. Ultimate flagellomere 88-118 $\mu \mathrm{m}$ long. Temporal setae 3-4, including 1-2 inner verticals and 2 outer verticals. Clypeus with 6-12 setae. Tentorium 115-130 $\mu \mathrm{m}$ long, 23-25 $\mu \mathrm{m}$ wide. Stipes 115-118 $\mu \mathrm{m}$ long, 4-5 $\mu \mathrm{m}$ wide. Palpomere lengths (in $\mu \mathrm{m}$ ): 48-53, 30-45, 48-60, 68-90, $123-$ 163. L: $5^{\text {th }} 3^{\text {rd }} 2.56-2.71$.

Wing (Fig. 8). Anal lobe normally developed. VR 1.17-1.19. Costal extension 30-38 $\mu \mathrm{m}$ long. Brachiolum with 1 seta. $\mathrm{R}$ with $1-3$ setae. Remaining veins bare. Squama with 2 setae.

Thorax (Fig. 9). Antepronotum with 4 lateral setae. Dorsocentrals 6-11; acrostichals 6-8, prealars 3 . Scutellum with 2-6 setae. Humeral pit moderately large, ovoid.

Legs. Spur of fore tibia $23-40 \mu \mathrm{m}$ long; spurs of mid tibia $13-18 \mu \mathrm{m}$ long and $12-15 \mu \mathrm{m}$ long; spurs of hind tibia $27-38 \mu \mathrm{m}$ and $13-15 \mu \mathrm{m}$ long. Hind tibial comb with 8-16 spines, 13-27 $\mu \mathrm{m}$ long. Width at apex of fore tibia $25-40 \mathrm{~mm}$, of mid tibia 25-38 mm, of hind tibia 23-40 mm. Lengths and proportions of legs as in Table 3.

Hypopygium (Figs 10-11). Anal point triangular, 20-28 $\mu \mathrm{m}$ long, with 4 lateral setae each side. Laterosernite IX with 2 setae. Phallapodeme 48-50 $\mu \mathrm{m}$ long. Transverse

Table 3. Lengths (in $\mu \mathrm{m}$ ) and proportions of legs of $R$. (P.) rotundus sp. $\mathrm{n}$.

\begin{tabular}{c|c|c|c}
\hline & $\mathbf{P}_{\mathbf{1}}$ & $\mathbf{P}_{\mathbf{2}}$ & $\mathbf{P}_{\mathbf{3}}$ \\
\hline $\mathrm{fe}$ & $330-490$ & $350-475$ & $310-445$ \\
\hline $\mathrm{ti}$ & $380-500$ & $310-485$ & $350-520$ \\
\hline $\mathrm{ta}_{1}$ & 218 & $135-240$ & $188-300$ \\
\hline $\mathrm{ta}_{2}$ & 153 & $80-135$ & $108-165$ \\
\hline $\mathrm{ta}_{3}$ & 120 & $65-110$ & $90-145$ \\
\hline $\mathrm{ta}_{4}$ & 75 & $35-50$ & $35-65$ \\
\hline $\mathrm{ta}_{5}$ & 50 & $40-50$ & $50-65$ \\
\hline $\mathrm{LR}$ & 0.57 & $0.44-0.49$ & $0.54-0.58$ \\
\hline $\mathrm{BV}$ & 2.33 & $3.48-3.61$ & $2.88-3.00$ \\
\hline $\mathrm{SV}$ & 3.26 & $4.00-4.89$ & $3.22-3.51$ \\
\hline $\mathrm{BR}$ & 2.67 & $2.00-2.80$ & $3.06-3.83$ \\
\hline
\end{tabular}



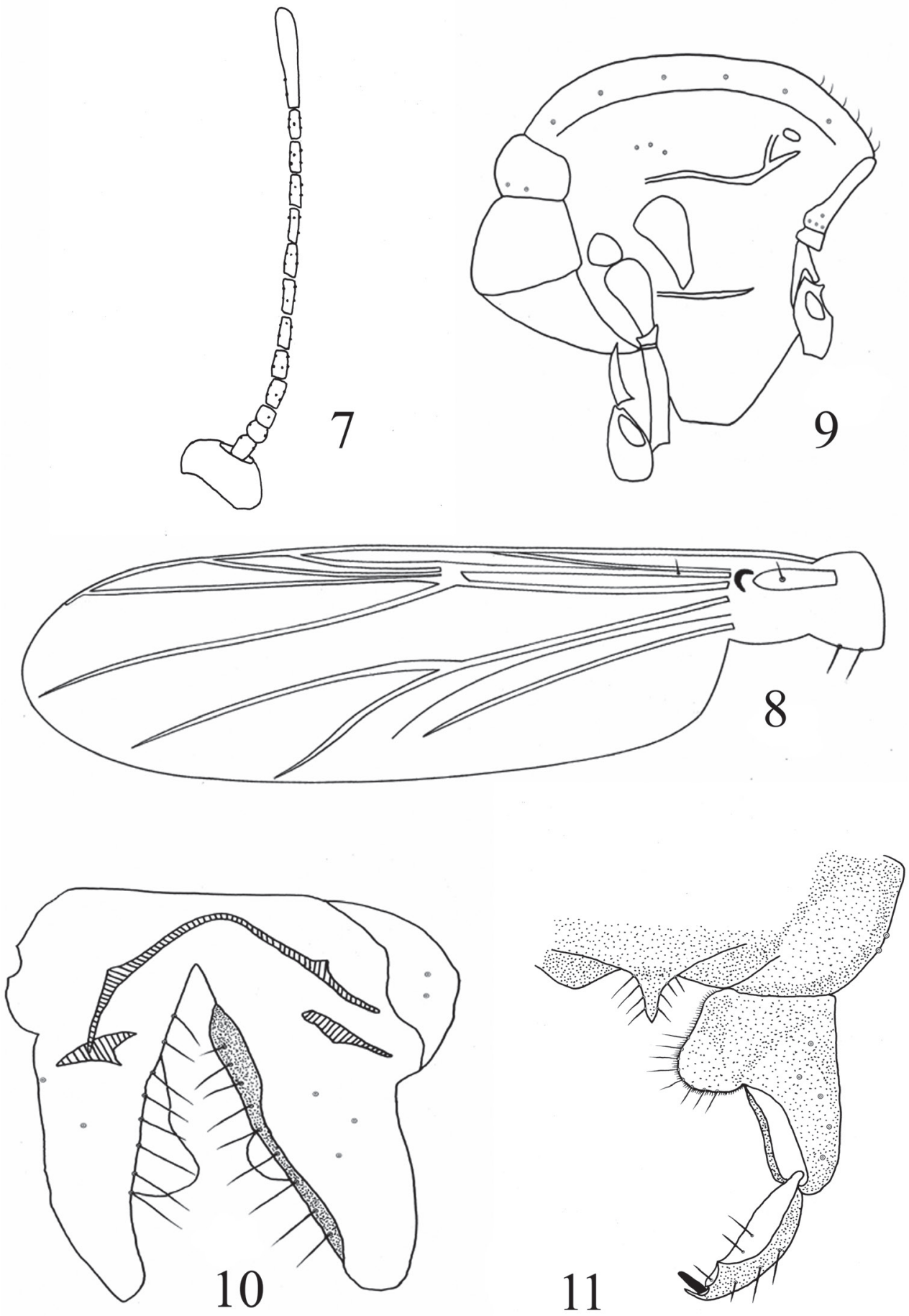

Figures 7-I I. Rheocricotopus (Psilocricotopus) rotundus sp. n., male. $\mathbf{7}$ antenna $\mathbf{8}$ wing $\mathbf{9}$ thorax $\mathbf{I} \mathbf{0}$ hypopygium (ventral view) II hypopygium (dorsal view). 
sternapodeme arcuate with normally oral projection, 33-40 $\mu \mathrm{m}$ long. Gonocoxite 125-135 $\mu \mathrm{m}$ long. Superior volsella rounded, plate-shaped and sclerotized, 28-38 $\mu \mathrm{m}$ long and 20-27 $\mu \mathrm{m}$ wide, covered with 8 short setae and a few microtrichia. Gonostylus 55-70 $\mu \mathrm{m}$ long, with distinct, triangular crista dorsalis. Megaseta 8-10 $\mu \mathrm{m}$ long. HR 1.93-2.27. HV 2.82-2.87.

Type material. Holotype: $\widehat{\delta}$ (BDN. G5A42), China, Zhejiang Province, Jinhua City, Pan'an County, Dapanshan National Nature Reserve, 28 $98^{\prime} 02^{\prime \prime} \mathrm{N}, 120^{\circ} 52^{\prime} 63^{\prime \prime E}$, 18.vii.2012, sweeping, Lin XL. Paratype (1): 1ð, Yunnan Province, Dali Bai Autonomous Prefecture, Eryuan County, Niujie Town, 2625'55"N, 9998'90"E, sweeping, Wang BX.

Etymology. The specific name is an adjective, from Latin rotundus, meaning rounded, referring to rounded superior volsella.

Remarks. The new species resembles $R$. (P.) notabilits Caspers, 1987 in the following combination of characters: low AR; humeral pit medium, ovoid; the shape of anal point. But the new species can be separated from latter species on the basis of following characters: (1) costal extension of the new species $(30-38 \mu \mathrm{m})$ much shorter than $R$. (P.) notabilits Caspers $(72 \mu \mathrm{m})$; (2) anal lobe of the new species developed, which reduced in $R$. (P.) notabilits Caspers; and (3) crista dorsalis of the new species distinct, triangular subapical, which pronounced, rounded in $R$. (P.) notabilits Caspers.

Female and immature stages unknown.

\section{Rheocricotopus (Psilocricotopus) serratus sp. $\mathrm{n}$.}

http://zoobank.org/D6FD7A2F-87EB-4513-A6D4-F122B14548DA

http://species-id.net/wiki/Rheocricotopus_serratus

Figs 12-15

Diagnosis. The adult male of the new species can be distinguished from known species of the species group and the genus by the following combination of characters: crista dorsalis sawtooth-shaped, hyaline, high as megaseta; high HR (2.61-3.42) and HV (3.40-5.00).

Description. Male imago $(\mathrm{n}=4)$

Total length 2.55-3.00, $2.73 \mathrm{~mm}$. Wing length 1.33-1.95, $1.69 \mathrm{~mm}$. Total length/ wing length 1.44-1.94, 1.64. Wing length/length of profemur 2.21-2.35, 2.27.

Coloration. Head and abdomen yellow, thorax dark brown.

Head. AR 0.71-0.78, 0.74. Ultimate flagellomere 285-355, $323 \mu \mathrm{m}$ long. Temporal setae $0-3,1$, including $0-1,1$ inner vertical; $0-2$, 1 outer vertical and $0-1,1$ postorbital. Clypeus with 9-12, 10 setae. Tentorium 140-153, $148 \mu \mathrm{m}$ long, 33-40, $38 \mu \mathrm{m}$ wide. Stipes 65-75, $68 \mu \mathrm{m}$ long, 3-7, $4 \mu \mathrm{m}$ wide. Palpomere lengths (in $\mu \mathrm{m}$ ): $35-70$, 47 ; 48-58, 53; 103-123, 114; 118-155, 141; 205-238, 219. L: $5^{\text {th }} / 3^{\text {rd }} 1.67-2.02,1.92$.

Wing (Fig. 12). Anal lobe slightly developed. VR 1.13-1.20, 1.17. Costal extension $50-88,71 \mu \mathrm{m}$ long. Brachiolum with 1 seta. $\mathrm{R}$ with 4-11, 7 setae. Remaining veins bare. Squama with 6-13, 9 setae. 

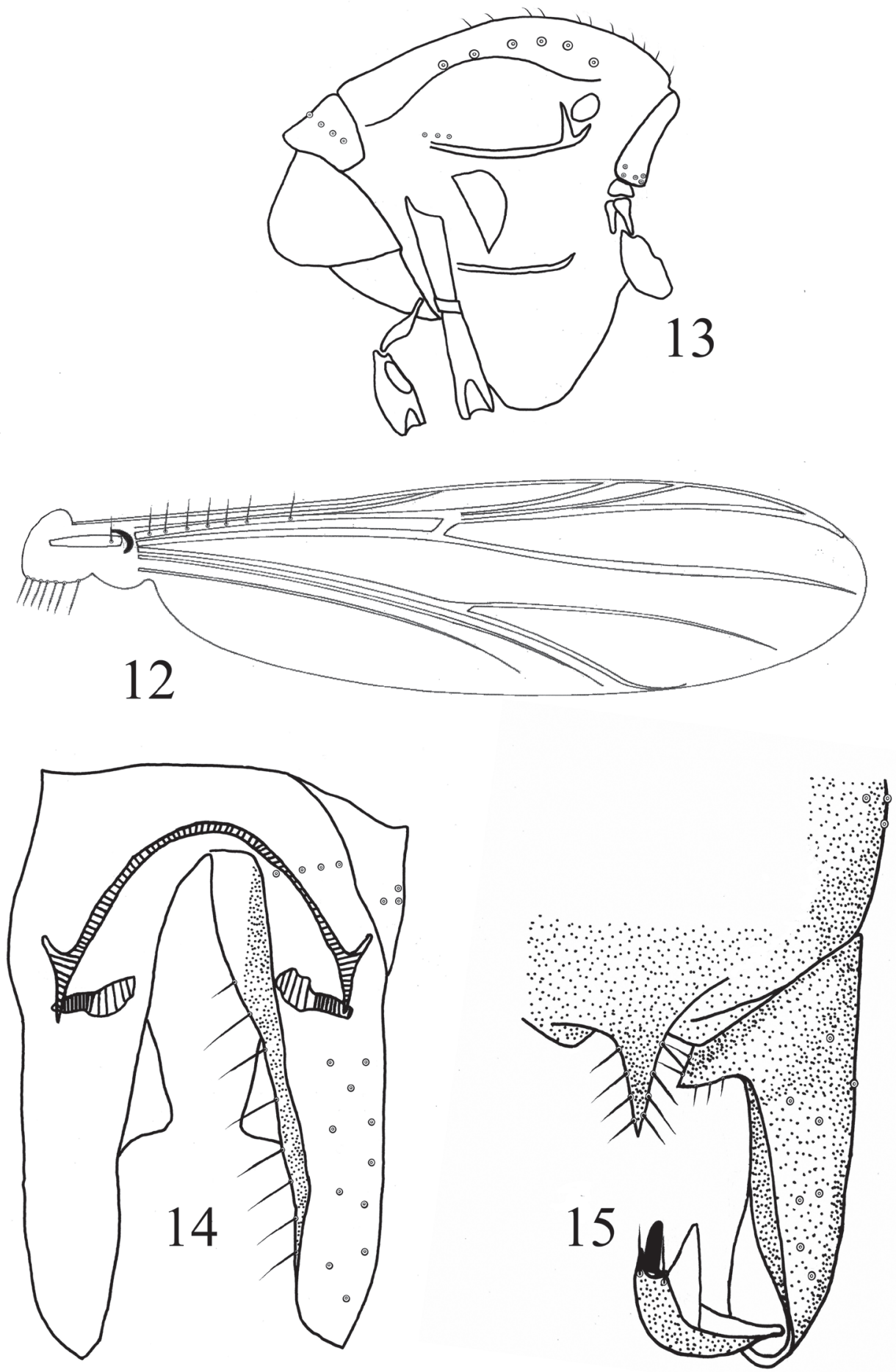

Figures I 2-I 5. Rheocricotopus (Psilocricotopus) serratus sp. n., male. $\mathbf{2} \mathbf{2}$ wing $\mathbf{I} \mathbf{3}$ thorax $\mathbf{I} \mathbf{4}$ hypopygium (ventral view) $\mathbf{5}$ hypopygium (dorsal view). 
Table 4. Lengths (in $\mu \mathrm{m}$ ) and proportions of legs of $R$. (P.) serratus sp. n.

\begin{tabular}{c|c|c|c}
\hline & $\mathrm{P}_{1}$ & $\mathrm{P}_{2}$ & $\mathrm{P}_{3}$ \\
\hline $\mathrm{fe}$ & $600-830,743$ & $570-800,700$ & $600-780,715$ \\
\hline $\mathrm{ti}$ & $650-810,760$ & $660-720,698$ & $660-850,783$ \\
\hline $\mathrm{ta}_{1}$ & $520-780,640$ & $310-410,380$ & $380-530,475$ \\
\hline $\mathrm{ta}_{2}$ & $290-390,350$ & $150-200,183$ & $200-270,253$ \\
\hline $\mathrm{ta}_{3}$ & $205-270,250$ & $100-140,125$ & $160-220,198$ \\
\hline $\mathrm{ta}_{4}$ & $155-200,181$ & $55-80,71$ & $90-120,105$ \\
\hline $\mathrm{ta}_{5}$ & $90-100,95$ & $60-80,73$ & $70-90,83$ \\
\hline $\mathrm{LR}$ & $0.78-0.96,0.84$ & $0.47-0.58,0.54$ & $0.58-0.63,0.61$ \\
\hline $\mathrm{BV}$ & $2.39-2.55,2.44$ & $3.71-4.22,3.96$ & $2.17-3.04,2.81$ \\
\hline $\mathrm{SV}$ & $1.95-2.61,2.37$ & $3.56-3.97,3.69$ & $2.92-3.32,3.17$ \\
\hline $\mathrm{BR}$ & $1.75-2.11,1.91$ & $2.10-2.22,2.19$ & $2.40-3.55,2.99$ \\
\hline
\end{tabular}

Thorax (Fig. 13). Antepronotum with 1-7, 4 lateral setae. Dorsocentrals 6-9, 7; acrostichals 9-15, 12; prealars 3. Scutellum with 4-6, 5 setae. Humeral pit moderately large, egg-shaped.

Legs. Spur of fore tibia 40-48, $44 \mu \mathrm{m}$ long; spurs of mid tibia 15-25, $19 \mu \mathrm{m}$ and 15-20, $17 \mu \mathrm{m}$ long; spurs of hind tibia 43-55, $48 \mu \mathrm{m}$ and 18-23, $19 \mu \mathrm{m}$ long. Hind tibial comb with 11-13, 12 spines, $30-50,40 \mu \mathrm{m}$ long. Width at apex of fore tibia $35-45,41 \mathrm{~mm}$, of mid tibia 36-45, $41 \mathrm{~mm}$, of hind tibia 38-45, $43 \mathrm{~mm}$. Lengths (in $\mu \mathrm{m})$ and proportions of legs as in Table 4.

Hypopygium (Figs 14-15). Anal point triangular, pointed distally, 33-50, $41 \mu \mathrm{m}$ long, 20-30, $24 \mu \mathrm{m}$ wide, with 4-5, 5 lateral setae each side. Laterosernite IX with 2-3, 3 setae. Phallapodeme 23-28, $25 \mu \mathrm{m}$ long. Transverse sternapodeme 18-33, 26 $\mu \mathrm{m}$ long. Gonocoxite 180-205, $195 \mu \mathrm{m}$ long. Superior volsella triangular, 35-43, 40 $\mu \mathrm{m}$ long, with 7-8, 8 setae. Gonostylus 60-75, $67 \mu \mathrm{m}$ long. Megaseta 13-15, $14 \mu \mathrm{m}$ long. Crista dorsalis sawtooth-shaped, hyaline, high as megaseta. HR 2.61-3.42, 2.91. HV 3.40-5.00, 4.09.

Type material. Holotype: $\widehat{\delta}$ (BDN. 10058), China, Yunnan Province, Dali Bai Autonomous Prefecture, Eryuan County, Niujie Town, 262 $25^{\prime} 55^{\prime \prime N}$, 99 $98^{\prime} 90^{\prime \prime E}$, light trap, Zhou CF. Paratypes (3): 10, Zhejiang Province, Qingyuan County, Bais-

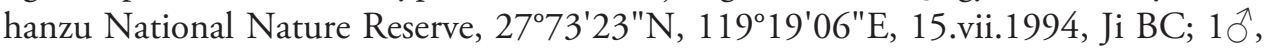
Tibet, Xigaze, Nielamu County, $27^{\circ} 98^{\prime} 73^{\prime \prime N}, 85^{\circ} 98^{\prime} 32^{\prime \prime E}, 21.9 .1987$, light trap,

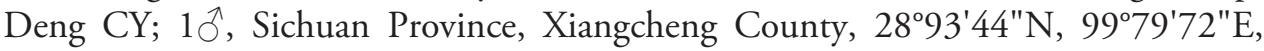
12.vi.1996, light trap, Wang XH.

Etymology. The specific name is an adjective, from Latin serratus, meaning sawtooth, referring to the sawtooth-shaped crista dorsalis.

Remarks. The new species resembles $R$. (P.) himalayensis Chaudhuri \& Sinharay, 1983 in the triangular anal point, but it can be separated from the latter species on the basis of following characters: (1) costal extension of the new species much longer (50-88 $\mu \mathrm{m})$, than $R$. (P.) himalayensis Chaudhuri \& Sinharay $(25 \mu \mathrm{m})$; (2) humeral 
pit in the new species medium, ovoid, which rounded in the latter species; (3) crista dorsalis sawtooth-shaped, hyaline in the new species, which moderately pronounced in the latter species.

Female and immature stages unknown.

Rheocricotopus (Psilocricotopus) taiwanensis Wang, Yan \& Maa, 2004 http://species-id.net/wiki/Rheocricotopus_taiwanensis

Rheocricotopus (Psilocricotopus) taiwanensis Wang, Yan \& Maa, 2004: 239, Ashe and O’Connor 2012: 567.

Specimens examined. Type material: Holotype, $\widehat{\partial}$, Taiwan Province, Taipei City, Guandu, Wetland, 2511'56"N, 121 ${ }^{\circ} 47^{\prime} 14^{\prime \prime E}$, 20.x.1988, sweeping, Maa CJ.

Diagnosis. The adult male can be separated from other members of the group by the following combination of characters: all veins of wing bare; low AR (0.71); squama with 3 setae; very pronounced crista dorsalis.

Distribution. China (Taiwan Province).

\section{Rheocricotopus (Psilocricotopus) valgus Chaudhuri \& Sinharay, 1983}

http://species-id.net/wiki/Rheocricotopus_valgus

Rheocricotopus valgus Chaudhuri \& Sinharay, 1983: 402.

Rheocricotopus (Psilocricotopus) valgus Ashe \& O'Connor, 2012: 568.

Specimens examined. $1 \delta^{\Uparrow}$, Guangdong Province, Fengkai County, Heishiding, $23^{\circ} 30^{\prime} 02^{\prime \prime} \mathrm{N}, 111^{\circ} 55^{\prime} 01^{\prime} \mathrm{E}, 20 . i v .1988$, sweeping, Wang XH; $1{ }^{\top}$, Guangxi Province,

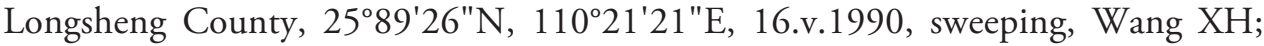
$10^{\top}$, Guangxi Province, Jinxiu County, $24^{\circ} 14^{\prime} 00^{\prime \prime N}, 110^{\circ} 19^{\prime} 00^{\prime} \mathrm{E}, 1 . v i .1990$, light

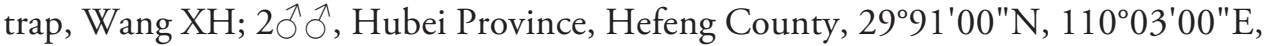
16.vii.1999, light trap, Ji BC; $10^{\Uparrow}$, Hubei Province, Xianfeng Mountain, $29^{\circ} 70^{\prime} 00^{\prime \prime} \mathrm{N}$, $119^{\circ} 14^{\prime} 00 " E$, 25.vii.1999, sweeping, Ji BC; 1ð, Zhejiang Province, Lishui City,

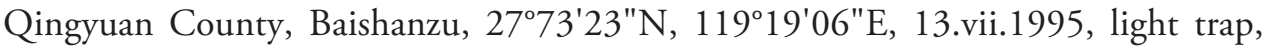
Ji BC; $4 \widehat{\jmath}$, Zhejiang Province, Lishui City, Qingyuan County, Baishanzu National Nature Reserve, $27^{\circ} 73^{\prime} 23 " \mathrm{~N}, 19^{\circ} 19^{\prime} 06^{\prime \prime E}, 24 . v i i .2012$, light trap, Lin XL;

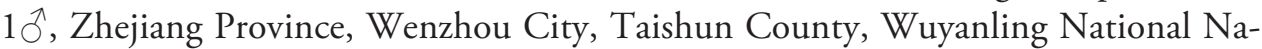
ture Reserve, $27^{\circ} 71^{\prime} 15^{\prime \prime} \mathrm{N}, 19^{\circ} 64^{\prime} 64^{\prime \prime} \mathrm{E}$, 3.viii.2005, light trap, Ji BC; $10^{\wedge}$, Zhejiang

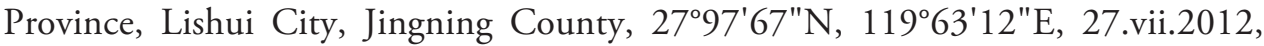
light trap, Lin XL.

Diagnosis. This species can be separated from other members of the group by the following combination of characters: R without seta; tergites I, II and anterior part of tergite V pale brown, tergites IV and VIII brown, anal point with 4-5 setae on each 
Table 5. Differences in the Chinese and Indian specimens of $R$. (P.) valgus

\begin{tabular}{c|c|c}
\hline & Chinese specimens & Indian specimens \\
\hline Squama & $5-8,7$ setae & 9 setae (average) \\
\hline HR & $1.98-2.15,2.08$ & 2.20 (average) \\
\hline HV & $2.88-3.20,3.03$ & 3.40 (average) \\
\hline
\end{tabular}

side and 1 seta at the base; gonocoxite with a prominent triangular basal lobe bearing 3-4 setae.

Remarks. The Chinese specimens generally agree with the original description by Chaudhuri and Sinharay (1983), though some measured differences between the Chinese specimens and those of Chaudhuri and Sinharay (1983) are shown in Table 5.

Distribution. China (Guangdong, Guangxi, Hubei and Zhejiang Provinces), India.

\section{Key to adult males of Rheocricotopus chalybeatus species group in China}

Anal lobe reduced 2

Anal lobe moderately or very developed. .5 Costa not beyond $\mathrm{R}_{4+}$ R. (P.) robacki (Beck \& Beck) Costa beyond $\mathrm{R}_{4+5}$ .. 3 Tergites I, II, IV yellow, tergite III with a brown circular area, others brown.. R. (P.) brochus sp. $\mathrm{n}$.

All tergites I-IX dark brown

R. (P.) imperfectus Makarchenko \& Makarchenko AR 0.25-0.29; superior volsella rounded $R$. $(P$.$) rotundus sp. \mathrm{n}$. AR $0.45-1.30$; superior volsella triangular .6 Humeral pit large, similar to the square .7 Humeral pit rounded or ellipsoid 8 Supraalars absent; crista dorsalis triangular 


\section{Acknowledgements}

Financial support from National Natural Science Foundation of China (NSFC, grant No. 30870329; 31272284; 31301908), Fauna of China (FY120100) and Sino-French Haihe IWRM Project (grant No. 2013DFA71340) are acknowledged with thanks. We are grateful to Bingchun Ji and Yufen Li who made parts of slide preparations.

\section{References}

Ashe P, O'Connor JP (2012) A world catalogue of Chironomidae (Diptera). Part 2B. Orthocladiinae. Irish Biogeographical Society \& National Museum of Ireland, Dublin, 558-575.

Beck WMJr, Beck EC (1964) New Chironomidae from Florida (Diptera). Florida Entomologist 47(3): 204. doi: 10.2307/3493325

Brundin L (1956) Zur Systematik der Orthocladiinae (Diptera, Chironomidae). Reporta from the Institute of Freshwater Research Drottningholm 37: 118.

Caspers N (1987) Rheocricotopus (Psilocricotopus) notabilis spec. nov. from Portugal (Diptera, Nematocera, Chironomidae).AquaticInsects9(3): 170. doi: 10.1080/01650428709361291

Chaudhuri PK, Sinharay DC (1983) A study on Orthocladiinae (Diptera, Chironomidae) of India. The genus Rheocricotopus Thienemann and Harnisch. Entomologcia Basiliensia 8: 398-407.

Cranston PS, Oliver DR, Sæther OA (1989) The adult males of Orthocladiinae (Diptera: Chironomidae) of the Holarctic region - Keys and diagnoses. In: Wiederholm T (Ed) Chironomidae of the Holarctic region. Keys and diagnoses. Part 3. Adult males. Entomological Scand Supplement (34): 165-352.

Edwards FW (1929) British non-biting midges (Diptera, Chironomidae). Transactions of the Entomological society of London 77: 279-430.

Goetghebuer M (1932) Diptères. Chironomidae IV. (Orthocladiinae, Corynoneurinae, Clunioninae, Diamesinae). Faune Entomologique Fran Aise 23: 1-204.

Goetghebuer M (1939) Etudes biospeologiques. XVI (1). Deux Chironomidae (Diptera) de Roumanie. Bulletin museums research Histoire Naturelle Belgique 15: 1-2.

Hazra N, Chaudhuri PK (2004) A new orthoclad species of Rheocricotopus Thienemann \& Harnisch (Diptera: Chironomidae) from the Darjeeling-Sikkim Himalyas in India. Animal Biodiversity and Conservation 27(2): 1-4.

Hirvenoja M (1973) Revision der Gattung Cricotopus van der Wulp und ihrer Verdandten (Diptera, Chironomidae). Annales Zoologici Fennici 10: 1-363.

Johannsen OA (1932) Orthocladiinae of the Malayan subregion of the Dutch East Indies. Archiv fir Hydrobiologie Supplement 9: 726-728.

Langton PH (1984) A key to pupal exuviae of British Chironomidae. Huntingdon, Cambridgeshire, $324 \mathrm{pp}$. 
Lehmann J (1969) Die europäischen Arten der Gattung Rheocricotopus Thien. und Harn. und drei neue Artvertreter dieser Gattung aus der Orientalis (Diptera, Chironomidae). Archiv fiir Hydrobiologie 66: 358-362.

Makarchenko EA, Makarchenko MA (2005) Chironomidae of the genus Rheocricotopus Thienemann ct Harnisch, 1932 (Diptera, Chironomidae, Othocladiinae) of the Russian Far East. Eurasian Entomological Journal 4(2): 126-134.

Makarchenko EA, Makarchenko MA (2011) Fauna and distribution of the Orthocladiinae of the Russian Far East. In: Wang X, Liu W (Ed) Proceedings of the 17th International Symposium on Chironomidae, 107-125.

Philippi RA (1865) Aufzählung der chilenischen Dipteren. Verh. Zool. Bot. Ges. Wien 15: 595-782.

Sæther OA (1969) Some Nearctic Podonominae, Diamesinae and Orthocladiinae (Diptera: Chironomidae). Bulletin of the Fishers Research Board of Canada 170: 1-154.

Sæther OA (1980) Glossary of Chironomid morphology terminology(Diptera: Chironomidae). Entomologica Scandinavica, Supplement 14: 1-51.

Sæther OA (1985) A review of the genus Rheocricotopus Thienemann \& Harnisch, 1932, with the description of three new species (Diptera, Chironomidae). Spixiana Supplement 11: 59-108.

Sasa M (1990) Studies on the chironomid midges (Diptera, Chironomidae) of the Nansei Islands, Southern Japan. Japanese Journal of Experimental Medicine 60(3): 127-129.

Sasa M (1991) Studies on the chironomids in Japan, 1991. Research Report from Toyama Prefectural Environment Pollution Research Center 1991: 72.

Sasa M, Suzuki H (2000) Studies on the chironomid species collected on Ishigaki and Iriomote Islands, Southwestern Japan. Tropical Medicine 42(1): 7.

Thienemann A, Harnisch O (1932) Chironomiden-Metamorphosen. IV. Die Gattung Cricotopus v. d. W. Zoologischer Anzeiger 99: 135-143.

Wang X (2000) A revised checklist of Chironomidae from China (Diptera). In: Hoffrichter O (Ed) Late 20th Century Research on Chironomidae: An Anthology from the 13th International Symposium on Chironomidae. Shaker Verlag, Aachen, 639 pp.

Wang X, Sxther OA (2001) Two new species of the orientalis group of Rheocricotopus (Psilocricotopus) from China (Diptera: Chironomidae). Hydrobiologa 444: 237-240. doi: 10.1023/A:1017548204492

Wang X, Zheng L (1989) Two new species of the genus Rheocricotopus from China (Diptera: Chironomidae). Entomotaxonomia 11(4): 311-313.

Wang X, Zheng L (1991) Notes on the genus Rheocricotopus from China (Diptera: Chironomidae). Acta Zootaxonomica Sinica 16 (1): 99-105.

Wang X, Yan CC, Mma CJ (2004) A New Rheocricotopus Thienemann \& Harnisch, 1932 (Diptera: Chironomidae) from Taiwan Province, China. Entomologia Sinica 11(3): 239. 